\title{
SAR: A NOVEL APPLICATION FOR FM-CW RADARS
}

\author{
J.J.M. de Wit, P. Hoogeboom \\ International Research Centre on Telecommunications-transmission and Radar \\ Delft University of Technology, Department of Electrical Engineering \\ Mekelweg 4, P.O. Box 5031, 2600 GA Delft, The Netherlands \\ Phone: +31 15 2781034, Fax: +31 15 2784046, E-mail: j.wit@irctr.tudelf.nl
}

\begin{abstract}
For small-scale earth observation applications, there is a special interest in low-cost, high-resolution imaging radars small enough to be operated from small, possibly unmanned, aircraft. The combination of the compactness of $F M-C W$ technology and the high resolution of SAR systems should result in such a small, cost-effective imaging radar.

In a new project the feasibility of FM-CW SAR is studied. SAR algorithms that take the typical characteristics of FM$C W$ signals into account will be developed. Furthermore, a low-cost demonstrator will be built. This demonstrator should prove the feasibility of FM-CW SAR and provide real test data to validate the processing algorithms and the design models. The demonstrator will be based on commercially available components. The system performance will be limited, but is sufficient to prove the feasibility of FM-CW SAR.
\end{abstract}

\section{INTRODUCTION}

There is a growing interest in imaging radars to be used in small-scale airborne earth observation applications. Examples of such small-scale applications are the monitoring of land use, or micro-farming, but one can also think of military applications, like the reconnaissance of enemy lines, or the detection and tracking of moving vehicles. Furthermore, there is a special interest in imaging radars with fast image generation capability in the field of civil crisis monitoring. Radar systems to be used for such applications should be of high-resolution, be cost-effective, and be small enough to be mounted on a small, possibly unmanned, airborne platform. However, existing imaging sensors, based on pulsed radar systems, are usually too heavy to be operated from small aircraft, or too expensive in daily use to be applied in smallscale civil applications.

Frequency modulated continuous wave (FM-CW) radars, on the other hand, are generally more compact and less expensive in day-to-day use than their pulsed counterparts. Furthermore, modern solid state microwave circuits can operate more efficiently in continuous wave mode.

Since radar resolution in azimuth direction may be enhanced applying synthetic aperture radar (SAR) techniques, the combination of SAR techniques and FM-CW technology should lead to a compact, cost-effective, high-resolution
imaging radar. For the operation of a compact FM-CW SAR a small, low-cost aircraft should be sufficient. This is another reason why FM-CW SAR could be cost-effective. But, to make FM-CW SAR a true competitor for small-scale civil applications, the operating costs should be lower than the costs of aerial photography.

Such a compact low-cost sensor would mean a breakthrough for remote sensing in small-scale applications. Nevertheless, SAR is an innovative application for FM-CW radars.

\section{PROJECT OVERVIEW}

A new research project is started to show the feasibility of FM-CW SAR technology in the field of airborne earth observation, and to design a low-cost, high-resolution system. During the project two main subjects will be addressed: A low-cost operation development of special signal processing algorithms.

should prove the feasibility of FM-CW SAR built, based on commercially available components. This demonstrator validation of the system feribity of FM-CW SAR, and provide real test data. These test data will be indispensable for the An important issue in SAR is the signal processing.

systems will not be rexisting SAR processing algorithms for pulsed SAR radars deliver signals in a pulse radars deliver signals in the time domain, whereas FM-CW of FM-CW radar signals into acncy domain. Therefore, special SAR algorithms, which take the typical characteristics test data obtained with the demonstrator In a later stage of the project the developm

In a later stage of the project the development of moving target indication techniques will be emphasised, and a study to real-time processing using commercial parallel computer components will he carried out. 


\section{SYSTEM DESCRIPTION}

The target system will operate in strip-mapping mode. To be of practical use the width of the imaged strip of land should be $1000 \mathrm{~m}$ or perhaps vider. The desired single-look resolution is $30 \mathrm{~cm}$ in range as well as in azimuth direction.

For the demonstrator it is assumed that the flying altitude will be around $250 \mathrm{~m}$ and that the platform velocity will be $150 \mathrm{~km} / \mathrm{hr}$. The minimun range will be $350 \mathrm{~min}$, the ground swath will be $500 \mathrm{~m}$, resulting in a maximum range of about $800 \mathrm{~m}$. The system characteristics are summarised in table 1.

The demonstrator will be based on a customised version of a low-cost FM-CW front-end, used in anti-collision car radars. The noise figure of this front-end is $8 \mathrm{~dB}$, and the system losses are $3 \mathrm{~dB}$. The antenna is a horn-lens antenna, providing a gain of $20 \mathrm{~dB}$.

A well-known problem in FM-CW radars is the leakage signal arriving at the receiver via the direct coupling between the transmitting and the receiving antennas. The phase noise associated with the leakage signal may mask echo signals if the antenna isolation is insufficient, or if the phase noise of the local oscillator is too high.

The slope of the phase noise is approximately $1 / f$, Wehner (1), where $f$ is the offset from the carrier frequency. Using a linear frequency modulation scheme, this offset frequency is proportional to range, Skolnik (2). Therefore, the phase noise is proportional to $1 / R$. However, for imaging radars the received signal is proportional to $1 / R^{3}$, Skolnik (2). So long-range weak scattering may be masked by the phase noise associated with a strong, short-range echo.

A first inventory already indicated that the sensitivity of the front-end to be used in the experimental system will be limited by phase noise rather than by thermal noise. Therefore, the leakage signal and reflections of the aircraft itself have to be carefully minimised. Regarding the phase noise leakage, it was already decided to reduce the ground swath to $500 \mathrm{~m}$, and also to relax the resolution specifications if necessary. Since the experimental system is merely used to show the feasibility of FM-CW SAR and to validate the design models, relaxing the specifications is not considered a problem.

\section{SYSTEM DESIGN MODELS}

The maximum SAR integration time is dependent upon the antenna beamwidth in azimuth direction and the platform velocity, and is given by:

$$
T_{S A R}=\frac{R_{f a r} \cdot \theta_{a}}{\nu}=0.64 \mathrm{~s}
$$

The corresponding synthetic aperture length is about $27 \mathrm{~m}$, leading to a theoretical azimuth resolution of $0.12 \mathrm{~m}$. In practice, this theoretical value will not be within reach since platform motion errors will limit the azimuth resolution.

The general form of the signal-to-noise ratio for radars is:

$$
S N R=\frac{P_{t} \cdot G^{2} \cdot \lambda^{2} \cdot \sigma}{(4 \pi)^{3} \cdot R^{4} \cdot k T_{0} B_{n} \cdot F_{n} \cdot L_{s}}
$$

where $\lambda$ is the carrier wavelength, $\sigma$ is the radar cross section (RCS) of the target, $k$ is Boltzmann's constant, $T_{0}=290 \mathrm{~K}$ is the standard temperature, and $B_{n}$ is the noise bandwidth. After determination of the beat frequency, the noise bandwidth $B_{n}$ is equal to the $P R F$, Griffiths (3).

However, in imaging radar applications the clutter-to-noise ratio $(C N R)$ is at least as important as the $S N R$, since one is mainly interested in the background. The $C N R$ can be calculated by replacing $\sigma$ in equation (2) by the clutter backscatter coefficient of the illuminated surface $\sigma_{\text {clutter }}$. After determination of the beat frequency the range resolution is already formed. Therefore, $\sigma_{\text {clutter }}$ should be calculated using a single resolution cell, rather than the entire swath width:

$$
\sigma_{\text {clurter }}=\Delta r_{r, \text { ground }} \cdot \Delta r_{a} \cdot \sigma_{0}=\Delta r_{r, \text { ground }} \cdot \theta_{a} \cdot R \cdot \sigma_{0} \approx-3 \mathrm{dBm}^{2}
$$

where $\Delta r_{r, \text { ground }}$ is the range resolution projected on the ground, and $\sigma_{0}$ is the radar cross section per unit area of the illuminated cell. In these calculations $\sigma_{0}$ is set to $-12.4 \mathrm{~dB}$; that is the clutter backscatter coefficient per unit area for grasses at an incidence angle of $70^{\circ}$ from the normal, Ulaby and Dobson (4). Combining equations (2) and (3) leads to:

$$
C N R=\frac{P_{1} \cdot G^{2} \cdot \lambda^{2} \cdot \Delta r_{r, \text { ground }} \cdot \theta_{a} \cdot \sigma_{0}}{(4 \pi)^{3} \cdot R^{3} \cdot k T_{0} \cdot P R F \cdot F_{n} \cdot L_{s}} \approx-2 \mathrm{~dB}
$$

After full SAR integration the clutter backscatter coefficient can be written as: 


$$
\sigma_{S A R}=\Delta r_{r, \text { ground }} \cdot \frac{\lambda}{2 T_{S A R} \cdot v} \cdot R \cdot \sigma_{0} \approx-26 \mathrm{dBm}^{2}
$$

And finally the CNR after full SAR integration follows as, Levanon (5):

$$
C N R_{S A R}=C N R \cdot \frac{\lambda \cdot P R F}{2 v \cdot \theta_{a}} \approx 2.58 \mathrm{~dB}
$$

The CNR is very low, even after full SAR integration. Since exact figures for the phase noise and the antenna isolation are not known yet, the phase noise is left out in these clutter-to-noise ratio calculations. Hence, in practice the $C N R$ will be even a bit lower. However, this low CNR may not be a problem, since the signal processing algorithms can probably be validated using a couple of larger targets.

Range ambiguities are not to be expected, since the unambiguous range is much larger than the maximum range of interest. However, some azimuth ambiguities may occur. The unambiguous beamwidth in azimuth direction is:

$$
\theta_{a, u n}=\frac{c \cdot P R F}{2 v \cdot f_{c}} \approx 6^{\circ}
$$

where $c$ is the speed of light. At the edges of the unambiguous azimuth beamwidth the antenna gain is about $13.5 \mathrm{~dB}$ down. Hence, large targets may cause azimuth ambiguities. Especially if due to aircraft yaw the antenna beam is pointing away from the area of interest. In the experimental system some azimuth ambiguities are tolerated.

The minimum and maximum beat frequency are $f_{b, \min }=1.16 \mathrm{MHz}$ and $f_{b, \max }=2.62 \mathrm{MHz}$ respectively. So the width of the beat spectrum is about $1.5 \mathrm{MHz}$, the sampling rate is set to $3.5 \mathrm{MHz}$ (real samples). Using 12 bits to code each sample leads to a bit rate of $5 \mathrm{Mbytes} / \mathrm{s}$. Regarding this data rate, the data acquisition chain can be implemented using off-the-shelf $\mathrm{A} / \mathrm{D}$ computer cards.

\section{SIGNAL PROCESSING}

The main purpose of SAR signal processing is to compress the raw data in range as well as in azimuth direction. Furthermore, the processing algorithms should correct motion errors, and typical SAR data features as range walk. To investigate the influence of the system parameters, for example the antenna pattern, motion errors, and platform velocity, upon the resolution, a computer simulation is being developed.

In FM-CW radars the received signal is mixed with a portion of the transmitted signal, producing a difference or beat frequency. For linear modulation schemes this beat frequency is proportional to range, Skolnik (2). If the radar beam is filled with several targets a beat spectrum will be produced after mixing. This beat spectrum can be determined by performing a Fourier transform on the time domain signal.

In SAR a large azimuth antenna aperture is synthesised by making use of the motion of the radar platform, Levanon (5). Due to the motion of the radar platform the range will differ from sweep to sweep, introducing a phase shift from sweep to sweep. After correction of this phase shift, the data can be compressed by performing a Fourier transform in azimuth direction, Soumekh (6).

Figure 1 shows the first results of the FM-CW SAR simulation for a single point target. Amplitude and noise factors, such as the antenna pattern and motion errors, are not included yet. First a Fourier transform is performed on each sweep to determine the beat spectrum, in the process the range resolution is formed. Figure 1a shows the range compressed data. Subsequently, the phase shift is corrected and the data are compressed in azimuth direction. Panel $b$ shows the point target's response after azimuth compression. The range and azimuth resolutions obtained with the simulation agree very well with the theoretical resolutions. The next step will be to include distorting factors, like the antenna pattem, noise, motion errors, etc.

\section{CONCLUSIONS}

There is an increasing interest in small, low-cost, high-resolution imaging radars in the field of small-scale airborne earth observation applications. An FM-CW SAR system, combining compact FM-CW technology with high-resolution SAR techniques, should be a good candidate for such applications. Nevertheless, SAR is a novel application for FM-CW ratars in the field of airborne earth observation.

To investigate the feasibility of FM-CW SAR a small, low-cost demonstrator will be built. The demonstrator will be Lascat on coinmercially avaiiable componerits. A first inventory indicated that the sensitivity of the denonstrator is limited by phase noise leakage rather than by thermal noise. One way to decrease the phase noise leakage is to maximise 
the antenna isolation. For future higher performance systems, phase noise can be further reduced by applying a high quality oscillator in the radar.

The performance level of the system will be evaluated and compared to the system design models. The models will be further improved to ensole the design of a future higher performarice PM-CW SAR for operational application. The signal processing a'gorittas will be furiher developed with the aid of the computer simulation, and finally they will be validated using the experimental data measured by the demonstrator.

\section{ACKNOWLEDGEMENTS}

The project is carried out at the Delft University of Technology, in close co-operation with the TNO-Physics and Electronics Laboratory (TNO-FEL) in The Hague. In particular, TNO-FEL will assist in the airborne measurement experiments. Furthermore, the company doubleBW in Delft is involved in the project. DoubleBW will provide the parallel computer components for the real-time processing.

\section{REFERENCES}

Wehner, D.R., "High-resolution radar", Artech House, Boston, 1995

Skolnik, M.I., "Introduction to radar systems", McGraw-Hill Inc., London, 1980

Griffiths, H.D., "Conceptual design of an ultra high-resolution mm-wave synthetic aperture radar", Proc. IEEE National Radar Conference, 255-260, 1996

Ulaby, F.T., Dobson, M.C., "Handbook of radar scattering statistics for terrain", Artech House, Norwood, 1989

Levanon, N., "Radar principles", John Wiley, New York, 1988

Soumekh, M., "Fourier array imaging", Prentice Hall, Englewood Cliffs, 1994

TABLE 1. System characteristics for the demonstrator.

\begin{tabular}{|l|l|l|l|}
\hline FM-CW front-end specifications & SAR layout \\
\hline Carrier frequency $f_{c}$ & $35 \mathrm{GHz}$ & Resolution range $\Delta r_{r}$ & $30 \mathrm{~cm}$ \\
\hline Frequency sweep $\Delta f$ & $500 \mathrm{MHz}$ & Resolution azimuth $\Delta r_{a}$ & $30 \mathrm{~cm}$ \\
\hline Sweep repetition frequency $P R F$ & $1 \mathrm{kHz}$ sawtooth & Ground swath & $500 \mathrm{~m}$ \\
\hline Transmitted power $P_{l}$ & $20 \mathrm{dBm}$ & Near range $R_{\text {near }}$ & $350 \mathrm{~m}$ \\
\hline Beamwidth range $\theta_{r}$ & $27^{\circ}$ & Far range $R_{\text {far }}$ & $800 \mathrm{~m}$ \\
\hline Beamwidth azimuth $\theta_{a}$ & $2^{\circ}$ & Altitude $h$ & $250 \mathrm{~m}$ \\
\hline Antenna gain $G$ & $>20 \mathrm{~dB}$ & Platform velocity $\nu$ & $150 \mathrm{~km} / \mathrm{hr}$ \\
\hline Antenna isolation & 45 to $50 \mathrm{~dB}$ & & \\
\hline Receiver noise figure $F_{n}$ & $8 \mathrm{~dB}$ & & \\
\hline System losses $L_{s}$ & $5 \mathrm{~dB}$ & & \\
\hline
\end{tabular}

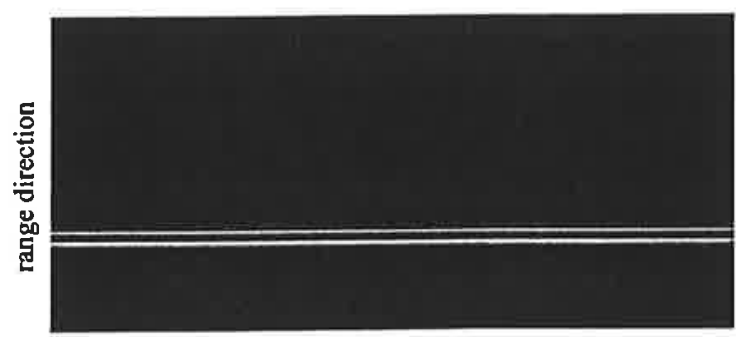

azimuth direction

(a)

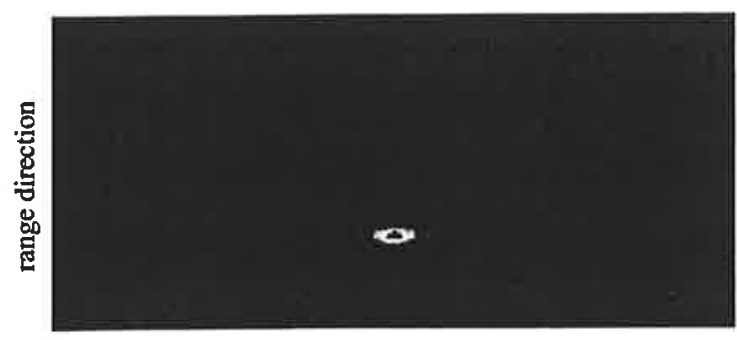

azimuth direction

(b)

FIGURE 1. Results of the simulation of the FM-CW SAR system for a single point target. Panel (a) shows the data after range compression, and panel (b) shows the data after azimuth compression. 


\section{1 \\ Conferente Proceedings}

\section{Volume 3 \\ Thursday 27th September 2001}

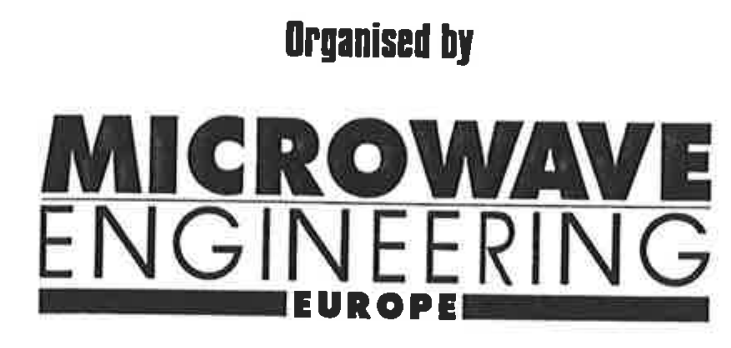

\section{on behalf of The European Mierowave Association}

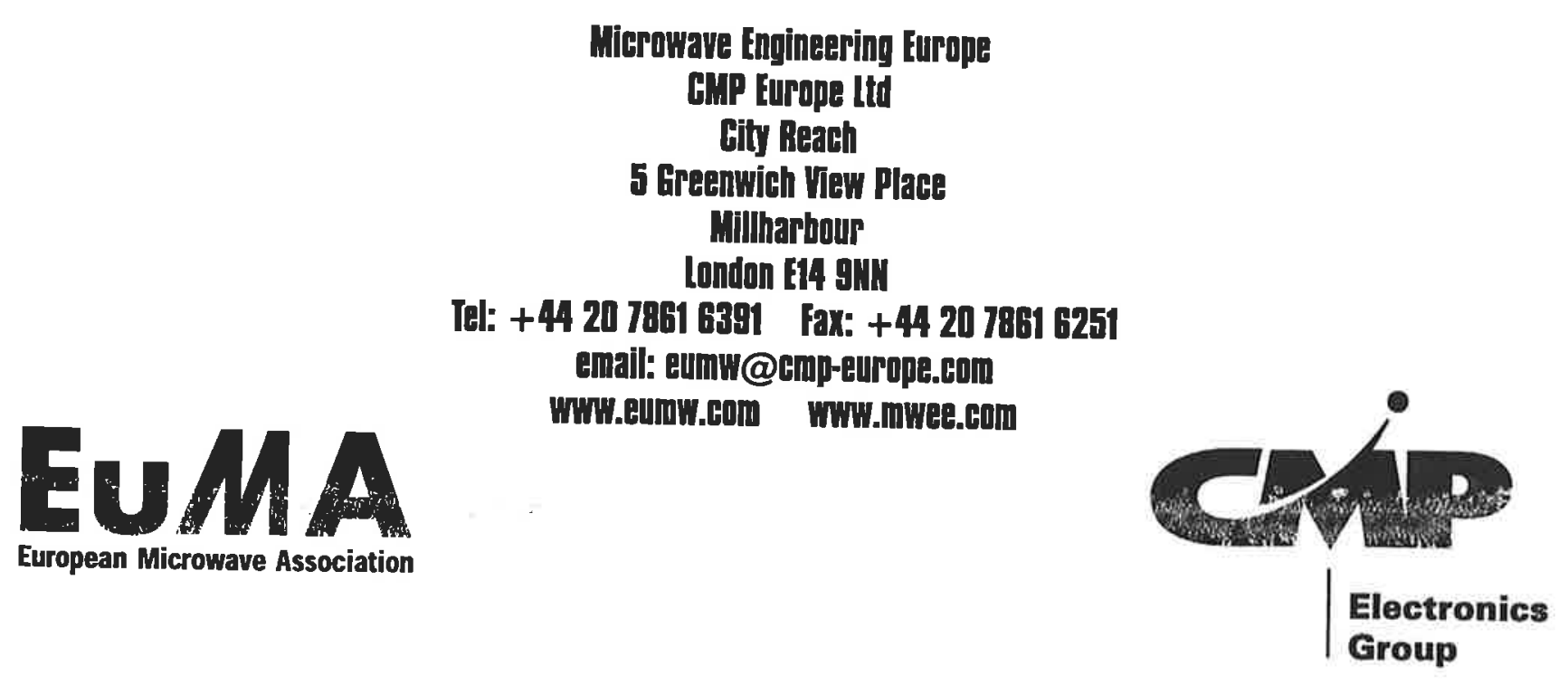

